\title{
DETECTION OF 8-HYDROXY-2-DEOXYGUANOSINE \& TOTAL ANTIOXIDANT CAPACITY IN SALIVA OF PATIENTS WITH ORAL PREMALIGNANT \& MALIGNANT LESIONS
}

\author{
Nayroz Abdelfattah Tarrad *, Sandy Shaaban Hassan * and Olfat Gamel Shaker**
}

\begin{abstract}
Background: the current study investigated the level of salivary 8-hydroxy-2-deoxyguanosine $\&$ total antioxidant capacity in oral premalignant and malignant lesions in order to determine their diagnostic value for the malignant patients.
\end{abstract}

Methods: A total of 120 subjects diagnosed with leukoplakia $(n=30)$, oral lichen planus $(n=30)$, oral squamous cell carcinoma $(n=30) \&$ healthy control subjects $(n=30)$ were included in this study. Salivary samples from all participants were collected. 8-hydroxy-2-deoxyguanosine was measured by enzyme linked immunosorbent assay technique and the level of total antioxidant capacity had been measured by means of photometric system.

Results: A significant higher salivary levels of 8-hydroxy-2-deoxyguanosine was observed in the group of oral squamous cell carcinoma in comparison to other groups. Comparing leukoplakia $\&$ oral lichen planus groups, no significant difference existed. Total antioxidant capacity showed its lowest value, with statistical significance, in oral squamous cell carcinoma patients. As between different grades of oral squamous cell carcinoma, significant difference had been registered in 8-hydroxy-2-deoxyguanosine between grade III and the other two grades while for total antioxidant only statistical significance existed between grade I and III.

Conclusion: Salivary 8-hydroxy-2-deoxyguanosine \& total antioxidant capacity are noteworthy diagnostic markers that can be utilized in diagnosis of oral squamous cell carcinoma.

KEYWORDS: Oral squamous cell carcinoma, saliva, 8-hydroxy-2-deoxyguinosine, total antioxidant capacity

\footnotetext{
* Lecturer of Oral Diagnosis, Oral Medicine and Periodontology, Department of Oral diagnosis, Oral Medicine, and Periodontology, Faculty of Dentistry, Fayoum University,

** Professor, Department of Biochemistry, Faculty of Medicine, Cairo University, Egypt.
} 


\section{INTRODUCTION}

Oral cancer is one of the most prevalent cancer types with oral squamous cell carcinoma (OSCC) representing over than $90 \%$ worldwide. ${ }^{1}$ OSCC usually stays unobserved until late stages which inversely affect the survival rate of patients. Therefore, to decrease its mortality rate, possible molecular diagnostic markers have been recently focused on to serve as predictors of oral malignancy. ${ }^{2}$ Oral leukoplakia and oral lichen planus (OLP) are considered among the precursors of malignant lesions affecting oral mucosa. ${ }^{3}$

Although oxygen is critical for living organisms to produce energy, it can be harmful through the formation of free radical compounds. Antioxidant systems are considered the protector against free radical toxicity. Oxidative stress results from free radical damage due to reactive oxygen species (ROS) over generation causing disequilibrium in oxidant \& antioxidant systems. ${ }^{4}$

ROS comprises hydroxyl radical (-OH), hydrogen peroxide $\left(\mathrm{H}_{2} \mathrm{O}_{2}\right)$ and superoxide $(\mathrm{O} 2) .{ }^{4}$ Antioxidant system is represented by catalase, superoxide dismutase, glutathione reductase $\&$ glutathione peroxidase. In addition, ascorbate, $\alpha$-tocopherol, glutathione, cysteine, thioredoxin \& vitamins. ${ }^{5,6}$ The hydroxyl radical $(\mathrm{HO} \bullet)$ is the main vital oxygenfree radical that can harm elementary biomolecules. These include proteins, membrane lipids \& DNA. Attachment of $\mathrm{HO} \bullet$ to DNA nucleobases, like guanine, causes synthesis of C8-hydroxyguanine or deoxyguanosine (8-hydroxy-2-deoxyguanosine) (8$\mathrm{OHdG}$ ) which is its nucleoside form. ${ }^{7,8}$

Total Antioxidant Capacity (TAC) is oxidants' quantity in moles which are neutralized by a liter of solution and is considered a marker used to assess the antioxidant potential of body fluids. ${ }^{9}$ As a result of oxidant-antioxidant system imbalance, massive oxidative damage to DNA caused by oxidants takes place, which consequently participates in many diseases such as aging and malignant tumors. ${ }^{10,11}$
To regain their normal function, enzymatical repair for damaged DNA takes place in the living cell, while unrepaired DNA can leads to mutations such as deletions, base substitution and strand fragmentation that can cause carcinogenesis.. ${ }^{12,13}$ 8-OHdG resulting from the oxidative damage to DNA elicited by ROS, leads to cell mutation and death. $^{14}$

To date, few studies linked 8-OHdG and TAC to oral squamous cell carcinoma and its different grades. Detection of molecular markers in saliva is superior to their detection in serum and other body fluids due to the fact of being an easy noninvasive tool. Consequently, the aim of our work was to investigate salivary $8-\mathrm{OHdG}$ and TAC in premalignant and malignant patients compared to normal subjects and to determine their diagnostic value for the malignant patients.

\section{SUBJECTS AND METHODS}

The current clinical trial was registered at ClinicalTrials.gov. ClinicalTrials.gov Identifier: NCT03830710.

\section{Study population}

120 subjects in total joined the present study. The clinically tested $\&$ confirmed subjects included:

Group A: 30 patients diagnosed with oral leukoplakia (16 females and 14 males; age range: 35-61 years).

Group B: 30 patients suffering from oral atrophic / erosive lichen planus (19 females and 11 males; age range: $35-60$ years).

Group C: 30 patients having oral squamous cell carcinoma with the tongue being the most commonly affected site and subdivided according to the histopathological grade into GI, GII and GIII (18 females and 12 males; age range: 35-62 years).

Group D: 30 age and gender matched individuals having no oral mucosal lesions acting as a control group (18 females and 12 males; age range: 35-63 years). 


\section{Ethical statement}

After describing the idea of the study, informed written consents from all the included patients and the control were obtained and signed.

\section{Inclusion criteria:}

All included subjects were assessed medically in conformity with the modified Cornell Medical index..$^{15}$ The patients were recruited from the diagnostic center, Faculty of Dentistry, Cairo University. All included individuals were not under any current medication and not suffering from any other systemic condition or any other oral mucosal disorder (based on clinical examination). The age of the study subjects was in the range of 35-63 years. The diagnosis of patients was based on clinical findings and was confirmed by histopathological examination. The histopathology should confirm the presence of dysplastic changes in the biopsy specimens of leukoplakia \& oral lichen planus patients in order to be included in the investigation.

\section{Exclusion Criteria}

The criteria to exclude subjects from the study included: (1) pregnant or lactating females; (2) subjects having systemic disorder or taking medications; (3) subjects who suffer from any other mucosal lesions; and (4) severe periodontal inflammation.

Comprehensive oral diagnosis was done for all participating individuals. Biopsy specimens were obtained from various lesions where a surgical double wedge incisional biopsy was carried out to a depth of about $2 \mathrm{~mm} .{ }^{16}$ Specimens were sent for histopathological examination to confirm the clinical diagnosis.

\section{Collection of salivary samples}

Whole unstimulated saliva had been collected from all participants by means of standard techniques defined by Navazesh. ${ }^{17}$ Subjects were asked to stop eating \& drinking for minimum half an hour prior to assessment. Collection of samples was carried out by requesting individuals to first swallow then tilt their head forward to expectorate saliva in a tube. This was done for 5 minutes. Obtained samples were then stored at $-20^{\circ} \mathrm{C}$ till assessed.

\section{Detection of salivary 8-OHdG:}

Saliva centrifugation has been done at $5000 \mathrm{x}$ $\mathrm{g}$ for 5 minutes. Removal of supernatant was done for determination of 8-hydroxy-2'-deoxyguanosine using an ELISA kit provided by BioVision, USA (Catalog \# K4160-100). 8-hydroxy-2'deoxyguanosine is an oxidized derivative of deoxyguanosine and is one of the major products of DNA oxidation.

Test principle: The ELISA is based on the competitive binding enzyme immunoassay technique. The microtiter plate provided in this kit has been pre-coated with an antibody specific to $8-\mathrm{OHdG}$. During the reaction, in the sample or standard competes with a fixed amount of biotinlabeled for sites on a pre-coated Monoclonal antibody specific to $8-\mathrm{OHdG}$. Excess conjugate and unbound sample or standard are washed from the plate. Next, Avidin conjugated to Horseradish Peroxidase (HRP) was added to each micro plate well and incubated. Then a tetramethylbenzidine (TMB) substrate solution was added to each well. The enzyme-substrate reaction was terminated by the addition of a sulphuric acid solution and the color change was measured spectrophotometrically at a wavelength of $450 \mathrm{~nm} \pm 2 \mathrm{~nm}$. The concentration in the samples was then determined by comparing the optical density (O.D) of the samples to the standard curve.

\section{Total antioxidative status/capacity (TAS/TAC) quantification in salivary samples}

Determination of TAS/TAC salivary levels was done using ImAnOx (TAS/TAC) Kit provided by Immun Diagnostik, Germany. It is a photometric test 
used for quantification of total antioxidative status/ capacity in serum, plasma \& further biological samples. The determination of the antioxidative capacity was carried out by the reaction of antioxidants in the sample with a defined amount of exogenously provided hydrogen peroxide ( $\mathrm{H} 2 \mathrm{O} 2)$. The antioxidants in the sample eliminate a certain amount of the provided $\mathrm{H} 2 \mathrm{O} 2$. The residual $\mathrm{H} 2 \mathrm{O} 2$ was determined photometrically by an enzymatic reaction which involves the conversion of TMB to a colored product. After addition of a stop solution, the samples were measured at $450 \mathrm{~nm}$ in a microtiter plate reader. The quantification was accomplished by the delivered calibrator. Difference between applied and measured peroxide concentration in a defined time period is proportional to the reactivity of the antioxidants of the sample (antioxidative capacity). Quantification was done by the enclosed calibrator.

\section{Statistical analysis}

Data obtained has been statistically analyzed to compare means of patient groups with the control group and within different patient subgroup using Tukey multiple comparisons of means. Data concerning OSCC group were analyzed with malignant grades (i.e. grade I, II and III according to histological findings). Correlation analysis of data with different grades was done. All statistical work was done using R statistical software version 3.3.3 $3^{18}$ using R Commander Package..$^{19,20}$

Receiver Operating Characteristic (ROC) curve has been made for determination of cut-off values of both markers to differentiate between included groups. Comparison of areas under the ROC curve (AUCs) was done by $z$-statistic. ROC curve analysis has been carried out using Version 11.3 MedCalc for Windows (MedCalc Software bvba).

\section{Estimation of Sample size}

The primary outcome in this study was sensitivity $\&$ specificity of $\mathrm{OHdG}$ to differentiate between
OSCC and control subjects. Based on Kaur et al. (2016) ${ }^{21}$ results, sensitivity and specificity values of $\mathrm{OHdG}$ were 80 and $78 \%$ respectively. At $5 \%$ alpha $(\alpha)$ level \& $20 \%$ Beta $(\beta)$ level ( $80 \%$ power), minimal sample size needed was 25 individuals in each group. To compensate for drop out, sample size was increased to 30 individuals in each group with total 120 individuals in the four groups. Epitools epidemiological calculators (http://epitools.ausvet. com.au) were used to calculate sample size.

\section{RESULTS}

The demographic data of all enrolled subjects and the salivary levels of $8-\mathrm{OHdG}$ and TAC (mean and standard deviation) are shown in table (1). The OSCC patients were further subdivided according to their histopathological findings into grades; GI $(n=8)$, GII $(n=14)$ and GIII $(n=8)$.

Tukey contrast was utilized to compare between the mean values of salivary 8 -OHdG level for each two groups. A high significant difference was registered when comparing OSCC group and controls, leukoplakia \& OLP groups in favor of the OSCC group. Comparing leukoplakia \& OLP groups revealed no significant difference. The healthy control group showed the lowest values.

As for salivary TAC level, OSCC group showed the lowest values, with significant difference compared to the other groups. On the contrary, the control group registered the highest levels of TAC with significant difference over the other included groups. Though, no significant difference was observed when comparing OLP with leukoplakia.

Considering the different grades among the OSCC group, statistical significant difference was detected between each grade $\&$ the other concerning 8-OHdG. There was only significant difference found between GI and GIII regarding the TAC level (table 1). 
Diagnostic accuracy of the two markers (ROC curve analysis)

\section{Differentiation between OSCC and control groups}

To differentiate between OSCC \& control groups, ROC curve analysis of both markers revealed a cut-off value of $2.2472 \mathrm{ng} / \mathrm{ml}$ for $\mathrm{OHdG}$ and $147.8 \mu \mathrm{mol} / \mathrm{l}$ for TAC. The diagnostic accuracy was $100 \%$ for both markers.

\section{Differentiation between leukoplakia and control groups}

For differentiation between Leukoplakia \& control groups, ROC curve analysis of both markers was done \& displayed in table (2), Figure (1A).

At $2.247 \mathrm{ng} / \mathrm{ml}$ cut-off value, OHdG showed 98.4 diagnostic accuracy while at a cut-off value of $320.4 \mu \mathrm{mol} / \mathrm{l}$; TAC showed $100 \%$ diagnostic accuracy for differentiation between leukoplakia and control groups.

Pair-wise comparison between areas under the ROC curve (AUC) of both markers exhibited no statistical significant difference among both markers $(z=0.359, P$-value $=0.720)$.

\section{Differentiation between OLP and control groups}

ROC curve analysis of the two markers for differentiation between OLP and control groups showed that at cut-off value of $2.2472 \mathrm{ng} / \mathrm{ml}$ for $\mathrm{OHdG}$ and $313.7 \mu \mathrm{mol} / \mathrm{l}$ for TAC; the diagnostic accuracy was $100 \%$ for both markers.

\section{Differentiation between OSCC and Leukoplakia groups}

For differentiation between OSCC \& leukoplakia groups, ROC curve analysis of both markers is presented in Table (2), Figure (1B).

At $5.06 \mathrm{ng} / \mathrm{ml}$ cut-off value; OHdG showed
96.7\% diagnostic accuracy while at a cut-off value of $147.8 \mu \mathrm{mol} / \mathrm{l}$; TAC showed $100 \%$ diagnostic accuracy for differentiation between OSCC and leukoplakia groups.

Pair-wise comparison between (AUC) of both markers presented no statistical significant difference among both markers $(z=1.146, P$-value $=0.252$ ).

\section{Differentiation between OSCC and OLP groups}

For differentiation between OSCC and OLP groups, ROC curve analysis of both markers is presented in Table (2), Figure (1C).

At cut-off value of $5.763 \mathrm{ng} / \mathrm{ml}$; OHdG showed $96.7 \%$ diagnostic accuracy while at a cut-off value of $147.8 \mu \mathrm{mol} / \mathrm{l}$; TAC showed $100 \%$ diagnostic accuracy for differentiation between OSCC and OLP groups.

Pair-wise comparison between (AUC) of both markers exhibited no statistical significant difference among both of them $(z=1.385, P$-value $=0.166)$.

\section{Differentiation between Leukoplakia and OLP groups}

For differentiation between leukoplakia \& OLP groups, ROC curve analysis of both markers is shown in Table (2), Figure (1D).

At cut-off value of $3.76 \mathrm{ng} / \mathrm{ml}$; OHdG showed $73.3 \%$ diagnostic accuracy while at a cut-off value of $303.7 \mu \mathrm{mol} / \mathrm{l}$; TAC showed $65 \%$ diagnostic accuracy for differentiation between leukoplakia and OLP groups.

Pair-wise comparison between (AUC) of both markers displayed no statistical significant difference between both markers $(z=1.601, P$-value $=0.109)$. 
TABLE (1): Results for comparisons (mean, standard deviation 'SD') between salivary markers' levels (8$\mathrm{OHdG}, \mathrm{TAC}$ ) and age among all included groups and different OSCC grades

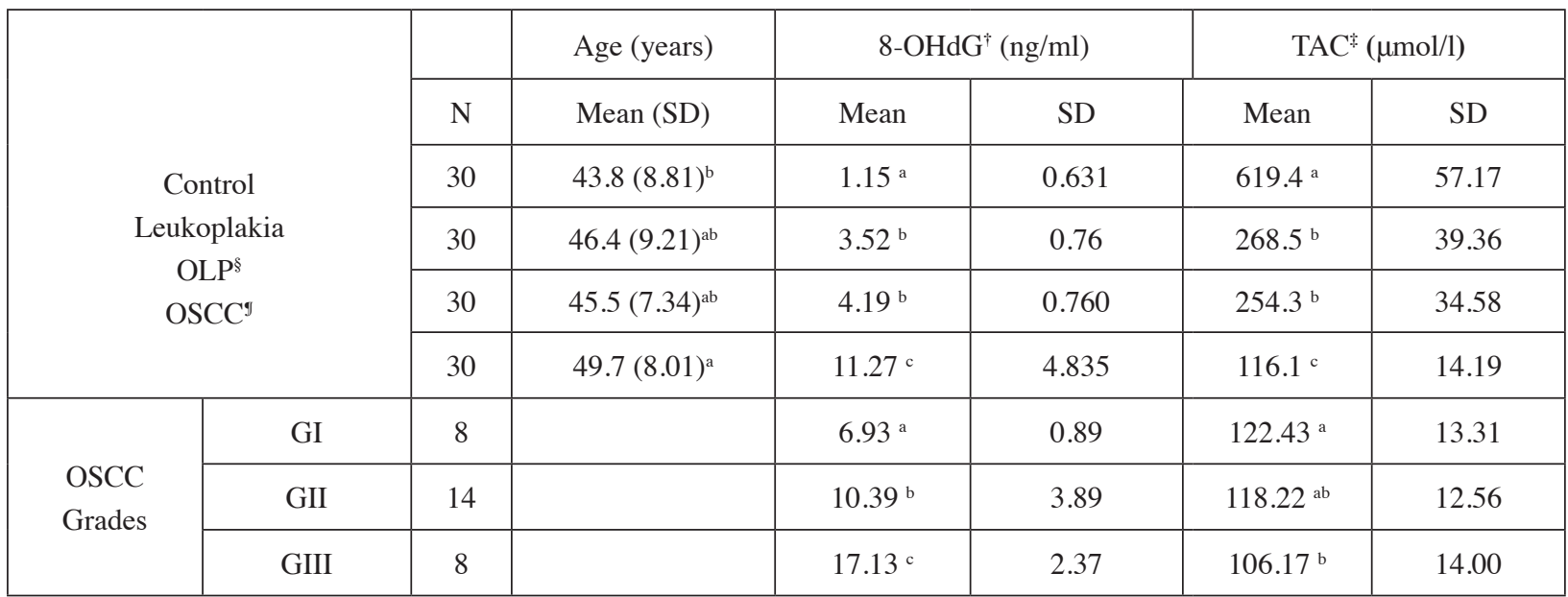

${ }^{\dagger} 8$ hydroxy-2-deoxyguanosine, ${ }^{\dagger}$ total antioxidant capacity, ${ }^{\prime}$ oral squamous cell carcinoma, ${ }^{8}$ Oral lichen planus. At 0.05 level, means with same letter within same column are not significantly different.

TABLE (2): Cut-off values of OHdG \& TAC, sensitivity, specificity, predictive values, diagnostic accuracy, Area under the ROC curve (AUC) \& 95\% confidence interval (CI) of (AUC) to differentiate between different groups

\begin{tabular}{|ccccccccccc|}
\hline \multirow{2}{*}{ Differentiation } & Marker & $\begin{array}{c}\text { Cut-off } \\
\text { value }\end{array}$ & $\begin{array}{c}\text { Sensitivity } \\
\%\end{array}$ & $\begin{array}{c}\text { Specificity } \\
\%\end{array}$ & +PV $\%$ & -PV \% & $\begin{array}{c}\text { Diagnostic } \\
\text { accuracy } \%\end{array}$ & AUC & $95 \%$ CI \\
\hline \multirow{2}{*}{ Leukoplakia/control } & OHdG & 2.247 & 96.7 & 100 & 100 & 96.8 & 98.4 & 0.998 & $0.936-1.000$ \\
& TAC & 320.4 & 100 & 100 & 100 & 100 & 100 & 1.000 & $0.940-1.000$ \\
\cline { 2 - 10 } & OHdG & 5.06 & 96.7 & 96.7 & 96.7 & 96.7 & 96.7 & 0.977 & $0.901-0.999$ \\
OSCC/Leukoplakia & TAC & 147.8 & 100 & 100 & 100 & 100 & 100 & 1.000 & $0.940-1.000$ \\
\cline { 2 - 10 } OSCC/OLP & OHdG & 5.763 & 93.3 & 100 & 100 & 93.7 & 96.7 & 0.967 & $0.885-0.996$ \\
& TAC & 147.8 & 100 & 100 & 100 & 100 & 100 & 1.000 & $0.940-1.000$ \\
\cline { 2 - 10 } & OHdG & 3.76 & 73.3 & 73.3 & 73.3 & 73.3 & 73.3 & 0.734 & $0.605-0.840$ \\
& TAC & 303.7 & 33.3 & 96.7 & 90.9 & 59.2 & 65 & 0.594 & $0.460-0.719$ \\
\hline
\end{tabular}

+PV: Positive Predictive Value, -PV: Negative Predictive Value 


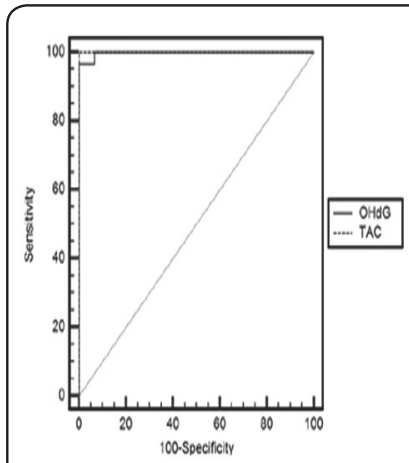

(A) Leukoplakia and control groups

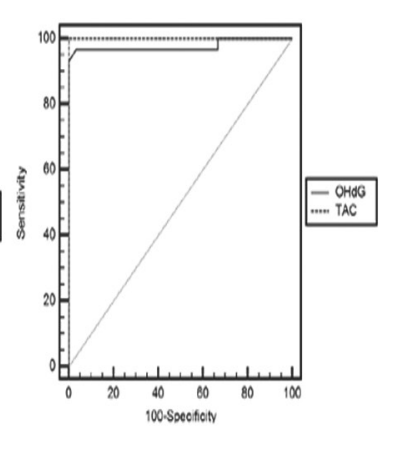

(B) OSCC \& Leukoplakia

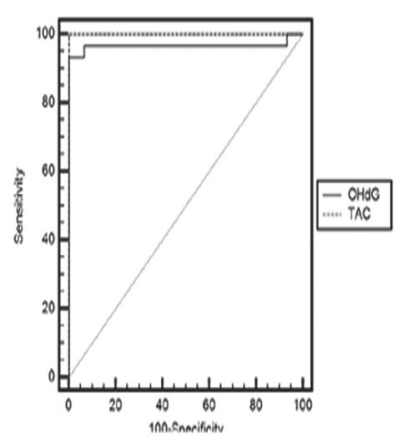

(C) OSCC \& OLP

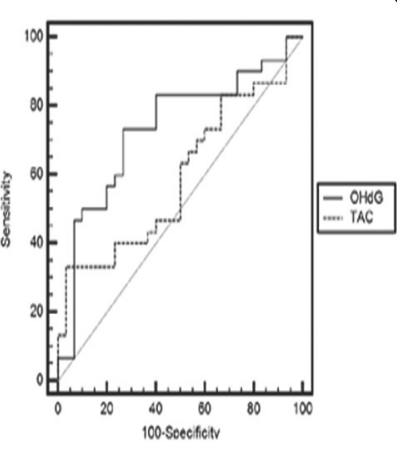

(D) Leukoplakia \& OLP

Fig. (1) ROC curves of 8-OHdG \&TAC to differentiate between different study groups

\section{DISCUSSION}

Oral squamous cell carcinoma is the sixth utmost prevalent cancer affecting the head and neck region worldwide. ${ }^{1}$ Oral precancerous lesions like oral lichen planus, leukoplakia \& oral submucous fibrosis usually precede oral malignant lesions. ${ }^{3}$ As mentioned in literature, the malignant transformation rate lies between 0.13 to $17.5 \%$ for leukoplakia. ${ }^{22}$ Consequently, to stop this transformation to a more aggressive lesion as well as decreasing the morbidity and mortality of malignant lesions, it is mandatory to early detect these oral premalignant lesions. ${ }^{23}$

Carcinogenesis mechanism is a multifactorial complicated process that includes molecular and cellular changes and is mediated by various endogenous and exogenous factors like ROS production. ${ }^{24}$

As guanosine is the most oxidized between the DNA nucleobases, $8-\mathrm{OHdG}$ is considered a biomarker of DNA oxidation destruction. ${ }^{8}$ Furthermore, $8-\mathrm{OHdG}$ has been extensively utilized as a carcinogenesis biomarker and was recently used for early detection of leukemia. ${ }^{25}$ TAC in saliva or urine was previously linked to many diseases like cancer, immune mediated and inflammatory disorders. ${ }^{26}$

Salivary disease biomarkers detection, besides being one of the most easily tools of diagnosis it is also a cost effective one in screening large number of population. Thus our aim was to investigate salivary levels of 8-OHdG and TAC in malignant and premalignant lesions to verify their diagnostic value for the malignant patients.

In our study the highest level of salivary $8-\mathrm{OHdG}$ was found with significant difference in OSCC compared to any other group supporting the fact that 8-OHdG plays a role in one of the mechanisms leading to malignant lesions through an increase in DNA oxidation by free radicals. ${ }^{27}$ This was in accordance with Kaur et al. ${ }^{21}$ who also reported significant increased levels of $8-\mathrm{OHdG}$ in OSCC. However, partially unlike the insignificant increased level of 8-OHdG among the OLP and leukoplakia groups shown in the present study, they reveled significantly high values in their premalignant groups, this might be owed to different population, used kits and number of subjects enrolled in both studies.

The lower levels of salivary antioxidant observed in OSCC group in our investigation in comparison to controls \& premalignant groups add another proof to the concept that antioxidants when demolished might have a participating effect in the pathological mechanisms taking place in malignant and premalignant lesions. ${ }^{21}$ Our observations concerning salivary TAC and $8-\mathrm{OHdG}$ in OSCC were in agreement with those obtained 
by Kumar et al. ${ }^{28}$ who showed elevated levels of $8-\mathrm{OHdG}$ and reduced TAC in their malignant groups in comparison to control subjects. Moreover, Pakfetrat et al ${ }^{29}$ observed lower levels of TAC in their malignant group compared to healthy controls but they used serum samples unlike salivary samples utilized in the present study.

Our results to some extent were in accordance to another investigation carried out by AghaHosseini et al..$^{30}$ as they found TAC salivary level significantly lower in OSCC group in comparison to controls although insignificant difference was observed between OSCC and OLP and also between OLP and the control which was contradicted to our findings. The same study also found similar results to our observations regarding salivary $8-\mathrm{OHdG}$ being statistically higher among OSCC compared to control and a significant difference among OLP $\&$ controls but unlike our results they observed no significant difference among OLP \& OSCC.

The overall results herein confirm the presence of a state of imbalance between oxidant/antioxidant systems, causing over production of $8-\mathrm{OHdG}$ and consumed TAC among patients suffering from OSCC.

Moreover, the ROC curve analysis that was performed in the current investigation revealed that both OHdG and TAC have high sensitivity and specificity and thus, could be used as diagnostic markers differentiating OSCC from healthy individuals and also from premalignant lesions.

To our knowledge, the present investigation is the first to assess the relationship between both salivary $8-\mathrm{OHdG}$ and TAC with tumor grades. Our observations showed a statistical significant difference concerning $8-\mathrm{OHdG}$ between each grade and the other while only statistical significant difference existed between grades I and III regarding the TAC values. Thus, future studies with larger sample size are required to confirm the current outcomes, in order to verify the utilization of salivary $8-\mathrm{OHdG}$ and TAC as a prognostic biomarker to follow up the disease progression.

In the light of our results, further investigations are recommended to assess salivary levels of 8-OHdG and TAC after treatment of malignant lesions to determine their prognostic value and to monitor treatment efficiency.

\section{ACKNOWLEDGEMENTS}

The authors acknowledge the contribution of Professor Abdel Fattah M Tarrad, professor of plant physiology and biochemistry, Agriculture Research Center, Cairo, Egypt for his contribution in the performed statistical analysis. Also, the authors are grateful for Dr Khaled Kerra (Biostatistician \& Specialist in Quality Management, Faculty of Dentistry, Misr International University, Cairo, Egypt) for conducting ROC curve analyses and the power analysis.

\section{REFERENCES}

1. Parkin DM, Bray F, Ferlay J, Pisani P. Global cancer statistics, 2002. CA Cancer J Clin 55: 74-108, 2005.

2. da Silva SD, Ferlito A, Takes RP, Brakenhoff RH, Valentin MD, et al. Advances and applications of oral cancer basic research. Oral Oncol 47: 783-791, 2011.

3. Gupta PC, Bhonsle RB, Murti PR, Daftary DK, Mehta FS Pindborg JJ. An epidemiological assessment of cancer risk of oral precancerous lesions in India with special reference to nodular leucoplakia. Cancer 63:2247-2252, 1989.

4. Thanan R, Oikawa S, Hiraku Y, Ohnishi S, Ma N, Pinlaor S, Yongvanit P, Kawanishi S, Murata M. Oxidative stress and its significant roles in neurodegenerative diseases and cancer. Int J Mol Sci.;16(1):193-217, 2014

5. Fridovich, I. Superoxide anion radical, superoxide dismutase, and related matters. The Journal of Biological Chemistry. 272, 18515-18517, 1997. doi:10.1074/ jbc. 272.30 .18515

6. Halliwell, B. and Gutteridge, J.M.C. Free radicals in biology and medicine. 3rd Edition, Clarendon Press, Oxford, 1999. 
7. Valko M, Izakovic M, Mazur M, Rhodes CJ, Telser J. Role of oxygen radicals in DNA damage and cancer incidence. Molecular and Cellular Biochemistry;266:37- 56, 2004.

8. Kasai H. Analysis of a form of oxidative DNA damage, 8-hydroxy-2deoxyguanosine, as a marker of cellular oxidative stress during carcinogenesis. Mutation Research; 387:147-163, 1997

9. Niki E. "Assessment of antioxidant capacity in vitro and in vivo," Free Radical Biology and Medicine, vol. 49, no. 4, pp. 503-515, 2010.

10. Poulsen HE, Prieme S, Loft S. Role of oxidative DNA damage in cancer initiation and promotion. European Journal of Cancer Prevention;7:9-16, 1998.

11. Beckman $\mathrm{KB}$, Ames $\mathrm{BN}$. The free radical theory of aging matures. Physiology Reviews;78:547-581, 1998.

12. Loft $S$, Poulsen HE. Cancer risk and oxidative DNA damage in man. Journal of Molecular Medicine;74:297-312, 1996.

13. Kawanishi S, Hiraku Y, Oikawa S. Mechanism of guaninespecific DNA damage by oxidative stress and its role in carcinogenesis and aging. Mutation Research;488:65-76, 2001 .

14. Meng X, Suo X, Ding W, Li X, Ding Y. Determination of 8-hydroxy-2'- deoxyguanosine derivatized with 4-chloro-7 nitrobenzofurazan in urine by CE-LIF. Electrophoresis.;35: 1873-9, 2014.

15. Kerr DA, Millard HD. Oral Diagnosis 2nd edition. CV. Mosby Company, Saint-Louis, pp. 17, 1965.

16. Colema DV, Evans DM, Baker R (Eds). Histological methods and type of biopsy. Biopsy pathology and cytology of the cervix, 2nd edition. Arnold Company, London, Pp.40; 1999.

17. Navazesh M. Methods for collecting saliva. Jam N.Y.Acad Sci. 20: 72-77; 1993.

18. R Core Team. R: A language and environment for statistical computing. R Foundation for Statistical Computing, Vienna, Austria, 2017. URL https://www.R-project.org/.

19. Fox, J. and Bouchet-Valat, M. Rcmdr: R Commander. R package version 2.3-1, 2016.

20. Fox, J. The R Commander: A Basic Statistics Graphical
User Interface to R. Journal of Statistical Software; 14(9): $1-42,2005$.

21. Kaur J, Politis C, Jacobs R. Salivary 8-hydroxy-2deoxyguanosine, malondialdehyde, vitamin $\mathrm{C}$, and vitamin $\mathrm{E}$ in oral pre-cancer and cancer: diagnostic value and free radical mechanism of action. Clin Oral Invest., 20:315-319, 2016.

22. Amagasa T, Yamashiro M, Uzawa N. Oral premalignant lesions: from a clinical perspective. Int J Clin Oncol, 16, $5-14,2011$

23. Calatayud AM, Estrada RB, Sebastián JBV, Jiménez OS, Barona CG. Oral leukoplakia: clinical, histopathologic, and molecular features and therapeutic approach: actas malignant disorder. J Clin Diagn Res.;8(8):1-4, 2014.

24. Klaunig JE, Xu Y, Isenberg JS, Bachowski S, Kolaja KL, Jiang $\mathrm{J}$ et al. The role of oxidative stress in chemical carcinogenesis. Environ Health Perspect 106:289-295, 1998.

25. Valavanidis A, Vlachogianni T, Fiotakis C. 8-Hydroxy-2' -deoxyguanosine (8-OHdG): a critical biomarker of oxidative stress and carcinogenesis. J Env Sci Health, Part C.; 27:120-39, 2009.

26. Peluso I and Raguzzini A. Salivary and urinary total antioxidant capacity as biomarkers of oxidative stress in humans. Patholog Res Int.; 2016:5480267, 2016.

27. Rai B, Kaur J, Jacobs R, Anand SC. Adenosine deaminase in saliva as a diagnostic marker of squamous cell carcinoma of tongue. Clin Oral Investig 15:347-349, 2011.

28. Kumar A, Pant MC, Singh HS, Khandelwal S. Determinants of oxidative stress and DNA damage (8OhdG) in squamous cell carcinoma of head and neck. Indian Journal of Cancer; (49)3: 309-315, 2012.

29. Pakfetrat A, Dalirsani Z, Hashemy SI, Ghazi A, Mostaan LV, Anvari K, Pour AM. Evaluation of serum levels of oxidized and reduced glutathione and total antioxidant capacity in patients with head and neck squamous cell carcinoma. J Can Res Ther;14:428-31, 2018.

30. Agha-Hosseini F, Mirzaii-Dizgah I, Farmanbar N, Abdollahi M. Oxidative stress status and DNA damage in saliva of human subjects with oral lichen planus and oral squamous cell carcinoma. J Oral Pathol Med; 41: 736-740, 2012 